Pacific Journal of Mathematics

REPRESENTATIONS BY ALGEBRAS OF SECTIONS OVER 


\title{
REPRESENTATIONS BY ALGEBRAS OF SECTIONS OVER BOOLEAN SPACES
}

\author{
Stephen D. Comer
}

\begin{abstract}
Every universal algebra is representable (in a trivial way) as the algebra of all continuous sections of many nonisomorphic sheaves (even over Boolean spaces). It is shown that on algebra, satisfying certain conditions specified below, can be represented as the algebra of all sections of a special kind of sheaf called a reduced sheaf. In addition, it is shown that the only reduced sheaf (up to isomorphism) whose sections represent an algebra satisfying the specified conditions is the one constructed in the standard way.
\end{abstract}

The construction used in [1], to represent a cylindric algebra as the algebra of all continuous sections of a reduced sheaf uniquely associated with the algebra, was intuitively patterned after the construction used in R. S. Pierce [3] to sectionally represent rings. This construction and the associated representation results are extended to a reasonable class of universal algebras including rings with unit and cylindric algebras. Whenever an algebra $\mathfrak{A}$ is represented as $\Gamma(X, S)$ with $X$ a Boolean space, $\mathfrak{A}$ is a subdirect product of the stalks $S_{x}$. An advantage of considering such representations for $(X, S)$ reduced is that, frequently in this case, the algebras $S_{x}$ have a simplier nature than $\mathfrak{i}$. For examples of this, see [1] and [3].

The universal algebras to be considered here are those algebras $A$ satisfying the following two properties.

(I) The set $\theta_{0}(\mathfrak{U})$ of all factor congruence relations of $\mathfrak{U}$ is a sublattice of $\theta(A)$ which is a Boolean algebra.

(II) Every congruence relation on $\mathfrak{U}$ generated by a proper Boolean ideal of $\theta_{0}(\mathfrak{X})$ is proper.

Condition (I) guarantees that the construction with yield a sheaf over a Boolean space and condition (II) guarantees that the stalks of this sheaf will be nontrivial when $\mathfrak{A}$ is nontrivial. Among the algebraic structures possessing properties (I), (II) are rings with identity and cylindric algebras. In addition it is well known that (I) holds whenever $\theta(\mathfrak{V})$ is distributive and it is trivial that (II) holds whenever the unit congruence relation ${ }^{2} A$ is compact in $\theta(\mathfrak{V})$. Thus, for example, lattices with 0 and 1 satisfy (I), (II).

$\mathrm{K}$. Keimel has independently obtained in [2] a result similar to Theorem 3.7. However, the algebras considered in [2] are more special than those considered here since they contain a one element subalgebra and it is assumed that outer direct products of these 
algebras correspond to inner direct products of subalgebras.

1. Preliminaries, We use standard universal algebra terminology; in particular, $\theta(\mathfrak{X})$ denotes the lattice of all congruence relations on $\mathfrak{A}, \phi \in \theta(\mathfrak{H})$ is a factor relation if there is a $\psi \in \theta(\mathfrak{l})$ such that $\phi \cap \psi=I d_{A}$ and $\phi \mid \psi={ }^{2} A$. The subset of $\theta(\mathfrak{U})$ consisting of all factor relations is denoted by $\theta_{0}(A)$.

A sheaf of algebras with similarity type $\tau$ is a triple $(X, S, \pi)$ where (i) $X$ and $S$ are topological spaces; (ii) $\pi: S \rightarrow X$ is a local homeomorphism of $S$ onto $X$; (iii) for each $x \in X, \pi^{-1}(x)=S_{x}$ is the universe of an algebra $\left\langle S_{x}, f_{i}^{x}\right\rangle_{i \in I}$ with similarity type $\tau$; and (iv) the natural operations induced on $S$ by the operations on each $S_{x}$ are continuous. We will suppress $\pi$ and denote a sheaf by $(X, S)$. More precisely, condition (iv) means the following. If $f_{i}^{x}$ is 0 -ary for all $x \in X$, then (iv) means that the map from $X$ to $S$ that associates $f_{i}^{x} \in S_{x}$ to each $x \in X$ is continuous. Now suppose $f_{i}^{x}$ is $n$-ary for all $x \in X$ where $n>0$. Let $n S=\left\{\left(y_{0}, \cdots, y_{n-1}\right) \in{ }^{n} S: \pi\left(y_{i}\right)=\pi\left(y_{j}\right)\right.$ for all $i, j<n\}$ with the relativized product topology. In this case, (iv) means that the map from $n S$ to $S$ that associates $f_{i}^{x}(y)$ to $y$ whenever $x=\pi\left(y_{0}\right)$ is continuous. The algebras $S_{x}$ are called the stalks of the sheaf $(X, S)$. We say that $(X, S)$ is trivial if $X$ is a one point space and the only stalk is a one element algebra.

A section of $(X, S)$ is a continuous mapping $\sigma: X \rightarrow S$ such that $\pi \sigma=I d_{X}$; the set of all sections of the sheaf $(X, S)$ is denoted by $\Gamma(X, S)$. If $(X, S)$ is a sheaf of algebras with type $\tau, \Gamma(X, S)$ can be made into an algebra with similarity type $\tau$ (also denoted by $\Gamma(X, S))$ by defining the operations pointwise. See [3] for the elementary basic properties of sheaves and sections. By a Boolean space we mean a compact, Hausdorff, zero dimensional space.

2. Reduced sheaves. In this section we define the notion of a reduced sheaf, give an equivalent characterization and show that $\Gamma(X, S)$ satisfies conditions (I), (II) if $(X, S)$ is reduced.

Suppose $(X, S)$ is a sheaf of algebras. For $\sigma, \tau \in \Gamma(X, S)$ the support of $(\sigma, \tau)$ is $|(\sigma, \tau)|=\{x \in X: \sigma(x) \neq \tau(x)\} ;|(\sigma, \tau)|$ is always a closed subset of $X$. For $\phi \in \theta(\Gamma(X, S))$ define $U_{\phi}=\cup\{|(\sigma, \tau)|:(\sigma, \tau) \in \phi\}$ and for a subset $U$ of $X$ let $\theta[U]=\left\{(\sigma, \tau) \in^{2} \Gamma(X, S):|(\sigma, \tau)| \leqq U\right\}$. $\theta[U] \in \theta(\Gamma(X, S))$ for every $U \subseteq X$. Define a function $\alpha$ on $\theta_{0}(\Gamma(X, S))$ by $\alpha(\dot{\phi})=U_{\dot{\phi}}$ whenever $\dot{\phi} \in \theta_{0}(\Gamma(X, S))$ and define a function $\beta$ on the Boolean algebra $(B A) \mathscr{C}$ of all clopen subsets of $X$ by $\beta(U)=\theta[U]$ whenever $U \in \mathscr{C}$.

Definition 2.1. A sheaf $(X, S)$ of algebras with similarity type $\tau$ is reduced if it is trivial or the following three conditions hold: 
(a) $X$ is a Boolean space;

(b) $S_{x}$ has at least two elements for all $x \in X$;

(c ) $\theta_{0}(\Gamma(X, S))$ is a sublattice of $\theta(\Gamma(X, S))$ and $\alpha$ is an isomorphism of $\theta_{0}(\Gamma(X, S))$ onto the $B A \mathscr{C}$ with inverse $\beta$.

Clearly, if $(X, S)$ is reduced, $\Gamma(X, S)$ satisfies condition (I) and, if also nontrivial, $X$ is the dual space of the $B A \theta_{0}(\Gamma(X, S))$.

For $\phi \in \theta(\Gamma(X, S))$ and $x \in X$ let $\dot{\phi}_{x}=\{(\sigma(x), \tau(x)):(\sigma, \tau) \in \phi\}$; if $X$ is a Boolean space $\phi_{x}$ is reflexive on $S_{x}$, symmetric and has the substitution property but is not a congruence relation on $S_{x}$ in general. The following result aids in verifying that a sheaf is reduced.

Proposition 2.2. Suppose a nontrivial sheaf $(X, S)$ satisfies (a), (b). Then $(X, S)$ is reduced if and only if

(i) for every $\dot{\phi} \in \theta_{0}(\Gamma(X, S))$ and $x \in X$, $\dot{\phi}_{x}=I d_{s_{x}}$ or $\dot{\phi}_{x}={ }^{2} S_{x}$;

(ii) for every $\dot{\phi} \in \theta_{0}(\Gamma(X, S)),\left\{x \in X: \dot{\rho}_{x}=I d_{s_{x}}\right\}$ is a clopen subset of $X$;

(iii) for every $\phi \in \theta_{0}(\Gamma(X, S)) \phi=\theta\left[U_{\phi}\right]$.

Proof. $\Rightarrow$ : Assume $(X, S)$ is reduced and nontrivial. (iii) is obvious. If $\phi \in \theta_{0}(\Gamma(X, S))$, then $\left\{x \in X: \phi_{x}=I d_{S_{x}}\right\}=X \sim U_{\phi}=\alpha(-\phi)$ is clopen so (ii) holds. Now suppose $\phi \in \theta_{0}(\Gamma(X, S)), x \in X$, and $\dot{\phi}_{x} \neq I d_{S_{x}}$. Then, by the above, $x \notin \alpha(-\phi)$ so $x \in \alpha(\phi)=\left\{x \in X:(-\phi)_{x}=I d_{S_{x}}\right\}$. For $a, b \in S_{x}$ choose $\sigma, \tau \in \Gamma(X, S)$ such that $\sigma(x)=a, \tau(x)=b . \quad(\sigma, \tau) \in \phi \mid-\dot{\phi}$ so $\sigma \dot{\phi} \mu(-\dot{\phi}) \tau$ for some $\mu \in \Gamma(X, S)$. Since $(-\phi)_{x}=I d_{S_{x}}, \mu(x)=b$; so $a \dot{\phi}_{x} b$. Hence $\dot{\phi}_{x}={ }^{2} S_{x}$ and (i) holds. $\sqsubset$ : We assume (a), (b), (i) - (iii), $(X, S)$ is nontrivial and verify (c).

(1) $\theta[N] \in \theta_{0}(\Gamma(X, S))$ whenever $N$ is a clopen subset of $X$ so $\beta: \mathscr{C} \rightarrow \theta_{0}(\Gamma(X, S))$.

It is easy to see that $\theta[N] \cap \theta[X \sim N]=I d_{\Gamma(X, S)}$. Now suppose $\sigma, \tau \in \Gamma(X, S)$ and define $\mu \in \Gamma(X, S)$ by $\mu=(\tau \mid N) \cup(\sigma \mid X \sim N)(\mu$ is a section by basic properties of sheaves). Then $\sigma \theta[N] \mu \theta[X \sim N] \tau$ so $\theta[N] \mid \theta[X \sim N]={ }^{2} \Gamma(X, S)$; hence (1) holds.

(2) If $\phi \in \theta_{0}(\Gamma(X, S)), U_{\phi}=\left\{x \in X: \phi_{x}={ }^{2} S_{x}\right\}$ and is clopen; thus, $\alpha: \theta_{0}(\Gamma(X, S)) \rightarrow \mathscr{C}$.

For suppose $x \in U_{\phi}$, i.e., $x \in|(\sigma, \tau)|$ for some $(\sigma, \tau) \in \phi$. Then $\sigma(x) \neq \tau(x)$ so $\dot{\phi}_{x}={ }^{2} S_{x}$ by (i); thus, $U_{\dot{\phi}}$ has the desired form and it is clopen by (i) and (ii).

(3) For $U \in \mathscr{C}, \alpha(\beta(U))=\left\{x \in X: \beta(U)_{x}={ }^{2} S_{x}\right\}=U$.

For $x \in U$ clopen there exist $\sigma, \tau \in \Gamma(X, S)$ such that $\sigma(x) \neq \tau(x)$ and $x \in|(\sigma, \tau)| \subseteq U$. Trivially, $\alpha(\beta(U)) \subseteq U$.

In view of (1)-(3), (iii) and the fact $\alpha$ and $\beta$ are order preserving, it is easily seen that $\alpha$ is a lattice isomorphism of $\theta_{0}(\Gamma(X, S))$ onto the $B A \mathscr{C}$ with inverse $\beta$. It remains to show that $\theta_{0}(\Gamma(X, S))$ is a 
sublattice of $\theta(\Gamma(X, S))$. Suppose $\phi, \psi \in \theta_{0}(\Gamma(X, S))$. Then $\phi+\psi \leqq$ $\beta(\alpha(\phi) \cup \alpha(\psi))$. For $(\sigma, \tau) \in \theta[\alpha(\phi) \cup \alpha(\psi)]$ let

$$
\mu=(\sigma \mid X \sim \alpha(\dot{\phi})) \cup(\tau \mid \alpha(\dot{\phi})) ;
$$

then $|(\sigma, \mu)| \leqq \alpha(\phi)$ and $|(\mu, \tau)| \leqq \alpha(\psi)$ since $|(\sigma, \tau)| \leqq \alpha(\phi) \cup \alpha(\psi)$. Hence $\sigma \phi \mu \psi \tau$; thus, $\phi+\psi=\beta(\alpha(\phi) \cup \alpha(\psi))=\dot{\phi} \mid \psi$ and so $\theta_{0}(\Gamma(X, S))$ is closed under + . It is easy to check that $\beta(\alpha(\phi) \cap \alpha(\psi))=\phi \cap \psi$ so $\theta_{0}(\Gamma(X, S))$ is closed under $\cap$. Hence (c) holds completing the proof of Proposition 2.2.

Suppose $\mathfrak{X}$ is an algebra satisfying condition (I). For an ideal $I$ of $\theta_{0}(\mathfrak{P})$ let $\bar{I}=\bigcup\{\theta: \theta \in I\}$ the congruence relation generated by $I$; for $\phi \in \theta(\mathfrak{I}) \quad I_{\phi}=\left\{\theta \in \theta_{0}(\mathfrak{I}): \theta \leqq \dot{\phi}\right\}$ is an ideal of $\theta_{0}(\mathfrak{Z})$. We say $\phi \in \theta(\mathfrak{U})$ is regular if $\bar{I}_{\phi}=\dot{\phi}$ and let $\theta^{R}(\mathfrak{U})$ denote the set of all regular congruences of $\mathfrak{N}$. It is easily seen that $\theta^{R}(\mathfrak{U})$ is a sublattice of $\theta(\mathfrak{I})$ and that the map $\gamma$, defined by $\gamma(\phi)=I_{\dot{\phi}}$ for $\phi \in \theta^{R}(\mathfrak{U})$, is a 1-1 map from $\theta^{R}(\mathfrak{U})$ into $I d\left(\theta_{0}(\mathfrak{U})\right)$, the lattice of all ideals of $\theta_{0}(\mathfrak{U})$. If $\gamma$ is onto $\operatorname{Id}\left(\theta_{0}(\mathfrak{X})\right)$, it is evident that it is also a lattice homomorphism and hence an isomorphism between $\theta^{R}(\mathfrak{R})$ and $I d\left(\theta_{0}(\mathfrak{A})\right)$. Condition (II) and $\gamma$ are linked in the following way.

Proposition 2.3. Assuming (I) holds for $\mathfrak{A}$, condition (II) holds if and only if $\gamma$ is onto (or equivalently, an isomorphism).

Proof. The nontrivial implication is to show that $\gamma$ is onto assuming (I), (II) hold. Suppose $I$ is a proper ideal of $\theta_{0}(\mathfrak{H})$ and let $\phi=\bar{I} . \quad \phi$ is regular so it is enough to show that $\gamma(\dot{\phi})=I$. Clearly, $I \subseteq I_{\phi}=\gamma(\phi)$. Now suppose $\psi \in \theta_{0}(\mathfrak{R}), \psi \in I_{\dot{\phi}}$, i.e., $\psi \leqq \phi$, and that $\psi \notin I$. Thus, the ideal $J$ of $\theta_{0}(\mathfrak{2})$ generated by $I \cup\{-\psi\}$ is proper. Let $M$ be a maximal $B A$ ideal of $\theta_{0}(\mathfrak{Q})$ extending $J$ (in particular, $M$ is proper). $\bar{M}$ is regular and $\bar{M} \supseteqq \phi$ since $M \supseteqq I$. By condition (II) $\bar{M} \neq{ }^{2} A$ so $\gamma(\bar{M}) \neq{ }^{2} A$. Since $\gamma(\overline{\bar{M}})$ is a proper $B A$ ideal containing $M$ which is maximal, $\gamma(\bar{M})=M$. Now $\psi \leqq \dot{\phi} \leqq \bar{M}$ and $\psi \in \theta_{0}(\mathfrak{O})$ so $\psi \in \gamma(\bar{M})=M$. On the other hand $-\psi \in J \subseteq M$ which contradicts the fact $M$ is proper. This contradiction shows that $\gamma(\dot{\phi}) \cong I$; hence the proof is complete.

Proposition 2.4. If $(X, S)$ is reduced, $\Gamma(X, S)$ satisfies (I), (II).

Proof. We have already observed that (I) holds in $\Gamma(X, S)$ when the sheaf is reduced; to verify (II) it is enough, by Proposition 2.3 , to assume $(X, S)$ is nontrivial and show $\gamma$ is onto. Suppose $I \in I d\left(\theta_{0}(\Gamma(X, S))\right)$ and $\dot{\phi} \in \theta_{0}(\Gamma(X, S))$ such that $\phi \in \gamma(\bar{I})$. Thus, $\alpha(\dot{\phi})$ 
is a clopen subset of $U_{I}=U\{\alpha(\psi): \psi \in I\}$. Since $\alpha(\psi)$ is clopen for $\psi \in I$, compactness implies there exist $\psi_{0}, \cdots, \psi_{n-1} \in I$ such that

$$
\alpha(\phi) \leqq \alpha\left(\psi_{0}\right) \cup \cdots \cup \alpha\left(\psi_{n-1}\right)=\alpha\left(\sum_{i<n} \psi_{i}\right) .
$$

Thus, $\dot{\rho} \leqq \sum_{i<n} \psi_{i} \in I$ so $\gamma(\bar{I})=I$; hence $\gamma$ is onto as desired.

For a nontrivial reduced sheaf $(X, S) \alpha$ induces an isomorphism $\alpha^{\prime}$ from $\operatorname{Id}\left(\theta_{0}(\Gamma(X, S))\right)$ onto the lattice of all open subsets of $X$. By Propositions 2.3, 2.4 $\alpha^{\prime} \circ \gamma$ is an isomorphism from $\theta^{R}(\Gamma(X, S)$ ) onto the lattice of all open subsets $X$. Observe that $\alpha^{\prime} \circ \gamma$ is just the map that associates $U_{\phi}$ with a regular congruence $\phi$; its inverse is just the map that associates $\theta[U]$ with an open subset $U$ of $X$.

3. Representation. In this section we represent an algebra satisfying (I), (II) as the algebra of all continuous sections of a reduced sheaf.

The trivial, i.e., one element, algebra $\mathfrak{A}$ satisfies (I), (II) and provides an uninteresting case. If we let $(X(\mathfrak{O}), S(\mathfrak{N}))$ be a trivial sheaf of algebras with the same similarity type as $\mathfrak{N}$, then $(X(\mathfrak{U}), S(\mathfrak{I}))$ is reduced and the obvious map $\xi_{\mathfrak{r}}$ is an isomorphism of $\mathfrak{U}$ onto $\Gamma(X(\mathfrak{U}), S(\mathfrak{U}))$.

Henceforth, we assume that $\mathfrak{A}$ has at least two elements and that properties (I), (II) hold. Our objective is to construct a sheaf $(X(\mathfrak{Y}), S(\mathfrak{U}))$ from $\mathfrak{Y}$. By (I) $\theta_{0}(\mathfrak{U})$ is a $B A$ so let $X(\mathfrak{U})$ be the Stone dual space of $\theta_{0}(\mathfrak{A})$, i.e., $X(\mathfrak{Q})$ is the set of all maximal ideals of $\theta_{0}(\mathfrak{A})$ with a topology given by a basis of clopen subsets consisting of all subsets of the form $\mathfrak{N}\left(\phi^{\prime}\right)=\{M \in X(\mathfrak{l}): \phi \notin M\}$ where $\phi \in \theta_{0}(\mathfrak{H}) . \quad X(\mathfrak{I})$ is a Boolean space.

Recall that $\bar{I}$ denotes the congruence relation on $\mathfrak{A}$ generated by the ideal $I$ of $\theta_{0}(\mathfrak{I})$. For $M \in X(\mathfrak{I})$ let $S(\mathfrak{U})_{M}=\mathfrak{U} / \bar{M}$. We set $S(\mathfrak{U})=$ $\cup\left\{S(\mathfrak{U})_{M}: M \in X(\mathfrak{I})\right\}$, the disjoint union of the $S(\mathfrak{I})_{M}{ }^{\prime}$ s. The map $\pi: S(\mathfrak{H}) \rightarrow X(\mathfrak{H})$ is defined in the obvious way.

In order to define the topology on $S(\mathfrak{U})$ we need some auxiliary functions. For $a \in A$ define $r_{a}: X(\mathfrak{Q}) \rightarrow S(\mathfrak{C})$ by $r_{a}(M)=a / \bar{M}$ for each $M \in X(\mathfrak{U})$. Now let $\mathscr{B}=\left\{r_{a}(\mathfrak{A}(\phi)): a \in A, \phi \in \theta_{0}(\mathfrak{I})\right\}$. In the following series of lemmas we will show that $(X(\mathfrak{U}), S(\mathfrak{U}))$ is a reduced sheaf with a basis for the topology on $S(\mathfrak{R})$ given by $\mathscr{B}$ and that $\mathfrak{U} \cong \Gamma(X(\mathfrak{Q}), S(\mathfrak{H}))$.

Lemma 3.1. For $a, b \in A$ and $M \in X(\mathfrak{H})$ such that $r_{a}(M)=r_{b}(M)$ there is $\dot{\rho} \in \theta_{0}(\mathfrak{U})$ such that $M \in \mathfrak{R}(\dot{\rho})$ and $r_{a}(N)=r_{b}(N)$ for all $N \in \mathfrak{N}(\dot{\phi})$.

Proof. Assume $a / \bar{M}=b / \bar{M}$. Since $\bar{M}=\bigcup\{\theta: \theta \in M\},(a, b) \in \theta$ for some $\theta \in M$. Choose $\phi=-\theta \in \theta_{0}(\mathfrak{Q})$. Then $M \in \mathscr{S}^{\prime}(\dot{\phi})$. For $N \in \mathfrak{R}(\dot{\phi})$, $(a, b) \in-\dot{\phi} \in N$ so $(a, b) \in \bar{N}$, i.e., $r_{a}(N)=r_{b}(N)$. 
Lemma 3.2. $\mathscr{B}$ is a basis for a topology on $S(\mathfrak{A})$.

Proof. Clearly $S(\mathfrak{A})=\cup \mathscr{B}$. Now suppose

$$
r_{a}(M) \in r_{b}\left(\mathscr{N}\left(\phi_{1}\right)\right) \cap r_{c}\left(\mathscr{N}\left(\phi_{2}\right)\right) .
$$

Thus, $M \in \mathscr{N}\left(\phi_{1}\right) \cap \mathscr{N}\left(\phi_{2}\right)$ and $a / \bar{M}=b / \bar{M}=c / \bar{M}$. By Lemma 3.1 there is a $\phi \in \theta_{0}(\mathfrak{X})$ such that $M \in \mathscr{N}(\phi)$ and $r_{b}(N)=r_{c}(N)$ for all $N \in \mathscr{N}(\phi)$. Thus, $\phi \cdot \phi_{1} \cdot \phi_{2} \in \theta_{0}(\mathfrak{Q})$ and, as easily seen,

$$
\begin{aligned}
r_{a}(M) & =r_{b}(M) \in r_{b}\left(\mathscr{N}\left(\phi \cdot \phi_{1} \cdot \phi_{2}\right)\right)=r_{c}\left(\mathscr{N}\left(\phi \cdot \phi_{1} \cdot \phi_{2}\right)\right) \\
& \leqq r_{b}\left(\mathscr{N}\left(\phi_{1}\right)\right) \cap r_{c}\left(\mathscr{N}\left(\phi_{2}\right)\right) .
\end{aligned}
$$

Hence $\mathscr{B}$ is a basis as claimed.

From 3.1, 3.2 it is clear that each $r_{a}(a \in A)$ is continuous.

LEMma 3.3. (X(I), $S(\mathfrak{Z}))$ is a sheaf of algebras.

Proof. From the preceding we know that $X(\mathfrak{U})$ and $S(\mathfrak{U})$ are topological spaces and that each stalk is an algebra with the same type as $\mathfrak{U}$. It is routine to check that $\pi$ is a local homeomorphism; in fact, it is enough to check that $\pi^{\prime}=\pi \mid r_{a}(\mathscr{N}(\phi))$ is a continuous map of $r_{a}(\mathscr{N}(\phi))$ onto $\mathscr{N}(\phi)$. It remains to establish the continuity of the natural operations on $S(\mathfrak{U})$ induced by corresponding operations on the stalk algebras $\mathfrak{X} / \bar{M}$. Suppose $f$ is an $n$-ary operation of this kind on $S(\mathfrak{U})$ where $n \in \omega \sim 1$. (We omit the easier case when $f$ is 0-ary.) Thus, $f: n S(\mathfrak{U}) \rightarrow S(\mathfrak{A})$ is defined, for $x \in n S(\mathfrak{U})$, by $f(x)=$ $f^{A / \bar{M}}(x)$ where $\pi\left(x_{0}\right)=M$. Suppose $r_{a}(\mathscr{N}(\dot{\phi}))$ is an open neighborhood of $f(x), M \in \mathscr{N}(\phi), x_{i}=a_{i} / \bar{M}$ where $a_{i} \in A(i<n)$, and consider $b=$ $f^{\mathfrak{2}}\left(a_{0}, \cdots, a_{n-1}\right)$. We have $r_{b}(M)=r_{a}(M)=f(x)$ so Lemma 3.1 implies there is $\psi \in \theta_{0}(\mathfrak{U})$ such that $r_{a}$ and $r_{b}$ agree on the neighborhood $\mathscr{N}(\psi)$ of $M$. Let $Y=n S(\mathfrak{U}) \cap\left[r_{a_{0}}(\mathscr{N}(\phi \cdot \psi)) \times \cdots \times r_{a_{n-1}}(\mathscr{N}(\phi \cdot \psi))\right] ; Y$ is an open neighborhood of $x$ in $n S(\mathfrak{U})$ and $f(Y) \subseteq r_{a}(\mathscr{N}(\phi))$. Consequently $f$ is continuous. It follows that $(X(\mathfrak{U}), S(\mathfrak{U}))$ is a sheaf.

The following universal algebraic result will be useful below. It is proved by a standard $B A$ argument.

LEMmA 3.4. If an algebra $\mathfrak{X}$ satisfies (I), and $I$ is a proper ideal of $\theta_{0} \mathfrak{A}$, then $\cap\{\bar{M}: \bar{I} \subseteq \bar{M}, M \in X(\mathfrak{U})\}=\bar{I}$.

We have seen (Lemma 3.3) that $(X(\mathfrak{U}), S(\mathfrak{U}))$ is a sheaf of algebras associated with $\mathfrak{U}$. Define $\xi_{\mathfrak{T}}$ by requiring $\xi_{\mathfrak{r}}(a)=r_{a}$ for each $a \in A$. From the remark following Lemma 3.2 we see that $\xi_{\mathfrak{q}}$ is a function from $\mathfrak{A}$ into $\Gamma(X(\mathfrak{U}), S(\mathfrak{U}))$. 
LEMMA 3.5. If $\mathfrak{Q}$ is an algebra satisfying conditions (I), (II), then $\xi_{\mathfrak{r}}$ is an isomorphism of $\mathfrak{X}$ onto $\Gamma(X(\mathfrak{U}), S(\mathfrak{U}))$.

Proof. We may suppose $\mathfrak{A}$ is nontrivial. It is routine to check that $\xi_{\mathfrak{r}}$ is a homomorphism. To show $\xi_{\mathfrak{r}}$ is $1-1$, assume $\xi_{\mathfrak{r}}(a)=\xi_{\mathfrak{r}}(b)$ where $a, b \in A$. Then $(a, b) \in \bar{M}$ for all $M \in X(\mathfrak{U})$ and hence Lemma 3.4 with $I=I d_{A}$ implies $a=b$. It remains to prove that $\xi_{\mathscr{q}}$ is onto. Suppose $\sigma \in \Gamma(X(\mathfrak{U}), S(\mathfrak{U}))$. By the construction of $S(\mathfrak{U})$, for each $M \in X(\mathfrak{U})$ there is an $a \in A$ such that $\sigma(M)=r_{a}(M) . \quad \sigma$ is continuous so there is a neighborhood $\mathscr{N}(\dot{\phi})$ of $M$ such that $\sigma$ and $r_{a}$ agree on $\mathscr{N}(\phi)$. Since this holds for each $M \in X(\mathfrak{H})$, the compactness and zero dimensionality for Boolean spaces implies that there exist $\phi_{0}, \cdots, \phi_{n-1} \in \theta_{0}(\mathfrak{U})$ and $a_{0}, \cdots, a_{n-1} \in A$ such that

(i ) $X(\mathfrak{i})=\mathrm{U}_{i<n} \mathscr{N}\left(\dot{\phi}_{i}\right)$;

(ii) $\mathscr{N}\left(\dot{\phi}_{i}\right) \cap \mathscr{N}\left(\dot{\phi}_{j}\right)=0$ whenever $i \neq j$; and

(iii) $\sigma$ agrees with $r_{a_{i}}$ on $\mathscr{N}\left(\dot{\phi}_{i}\right)$ for each $i<n$.

Since $\mathscr{N}\left(\dot{\phi}_{i}\right)=\left\{M \in X(\mathfrak{U}):-\dot{\phi}_{i} \in M\right\}$, (i) together with Lemma 3.4 implies that $\Pi_{i<n}-\dot{\phi}_{i}=I d_{A}$ and (ii) implies that $-\dot{\phi}_{i}+-\phi_{j}={ }^{2} A$ whenever $i \neq j$. This last fact implies $\left(\prod_{i \neq j}-\dot{\phi}_{j}\right) \mid-\dot{\phi}_{i}={ }^{2} A$ since $\theta_{0}(\mathfrak{U})$ is a $B A$. Applying a form of the Chinese Remainder Theorem (Cor. 1.6 of [4], p. 67) we conclude that the map

$$
x m\left(x /-\phi_{0}, \cdots, x /-\phi_{n-1}\right)
$$

gives an isomorphism of $\mathfrak{U}$ onto $P_{i<n} \mathfrak{U} /-\phi_{i}$. Hence, for the elements $a_{0}, \cdots, a_{n-1} \in A$ the above isomorphism shows there is an element $b \in A$ such that $b\left(-\phi_{i}\right) a_{i}$ for all $i<n$. It now follows that $\xi_{\mathscr{Y}}(b)=r_{b}=\sigma$; for if $N \in X(\mathfrak{U})$, say $N \in \mathscr{N}\left(\dot{\phi}_{j}\right)$ for some $j<n$, then $\sigma(N)=r_{a_{j}}(N)=$ $a_{j} / \bar{N}=b / \bar{N}=r_{b}(N)$ using the fact that $\left(b, a_{j}\right) \in-\phi_{j} \leqq \bar{N}$. We conclude that $\xi_{\mathfrak{r}}$ is onto completing the proof of the lemma.

We will show that the sheaf $(X(\mathfrak{U}), S(\mathfrak{Q}))$ constructed from $A$ is reduced by applying Proposition 2.2. Before doing this we need an observation about the relations $\phi_{M}=\{(\sigma(M), \tau(M)):(\sigma, \tau) \in \phi\}$, where $\phi \in \theta(\Gamma(X(\mathfrak{U}), S(\mathfrak{Y})))$ and $M \in X(\mathfrak{O})$, that are involved in the proposition. Let $\phi_{M}^{\prime}$ denote the transitive closure of $\phi_{M}$; it is a routine argument to show that $\phi_{M}^{\prime}$ is a congruence relation on $\mathfrak{U} / \bar{M}=S(\mathfrak{U})_{M}$ and that $\phi_{M}^{\prime}=\left(\xi_{\mathfrak{x}}^{-1}(\phi)+\bar{M}\right) / \bar{M}$, i.e., $\dot{\phi}_{M}^{\prime}$ is the congruence relation on $\mathfrak{X} / \bar{M}$ corresponding to the congruence relation $\xi_{\mathfrak{r}}^{-1}(\dot{\phi})+\bar{M}$ on $\mathfrak{X}$ under the correspondence induced by the canonical homomorphism of $\mathfrak{U}$ onto $\mathfrak{U} / \bar{M}$.

Lemma 3.6. If an algebra $\mathfrak{X}$ satisfies (I), (II), then (X(Y), $S(\mathfrak{X})$ ) is reduced. 
Proof. Assuming $\mathfrak{A}$ nontrivial conditions 1.1 (a), (b) are obvious so it suffices to verify properties (i), (ii), (iii) in Proposition 2.2.

(i) Suppose $\phi \in \theta_{0}(\Gamma(X(\mathfrak{U}), S(\mathfrak{U})))$ and $M \in X(\mathfrak{U}) . \quad \xi_{\mathfrak{r}}^{-1}(\dot{\phi}) \in \theta_{0}(\mathfrak{U})$ so either $\xi_{\mathfrak{\varkappa}}^{-1}(\dot{\phi}) \in M$ or $-\xi_{\mathfrak{\varkappa}}^{-1}(\phi) \in M$. In the first case

$$
\dot{\phi}_{M}^{\prime}=\left(\xi_{\mathfrak{r}}^{-1}(\phi)+\bar{M}\right) / \bar{M}=I d_{S_{M}} \text { so } \dot{\phi}_{M}=I d_{S_{M}} \text {. }
$$

It remains to show that $-\xi_{\mathfrak{\Re}}^{-1}(\phi) \in M$ implies $\phi_{M}={ }^{2} S_{M}$. Assume $-\xi_{\Re}^{-1}(\phi) \in M$ and $\left(r_{a}(M), r_{b}(M)\right) \in{ }^{2} S_{M} . \quad(a, b) \in{ }^{2} A=\xi_{\Re}^{-1}(\phi) \mid-\xi_{\Re}^{-1}(\phi) \quad$ so there is a $c \in A$ such that $(a, c) \in \xi_{\mathfrak{r}}^{-1}\left(\phi^{\prime}\right)$ and $(c, b) \in-\xi_{\mathfrak{r}}^{-1}\left(\phi^{\prime}\right) \leqq \bar{M}$. Therefore, $\quad\left(r_{a}, r_{c}\right) \in \phi$ and $c / \bar{M}=b / \bar{M} ;$ thus, $\quad\left(r_{a}(M), r_{b}(M)\right) \in \phi_{M L}$ as desired. Hence (i) holds.

(ii) Suppose $\phi \in \theta_{0}(\Gamma(X(\mathfrak{U}), S(\mathfrak{Y})))$. By the proof of (i) we see that $\left\{M \in X(\mathfrak{U}): \dot{\rho}_{M I}=I d_{S_{M}}\right\}=\left\{M \in X(\mathfrak{U}): \xi_{\mathfrak{q}}^{-1}(\dot{\phi}) \in M\right\}=\mathscr{N}\left(-\xi_{\mathfrak{r}}^{-1}(\dot{\phi})\right)$ is a clopen subset of $X(\mathfrak{i})$.

(iii) We always have $\phi \leqq \theta\left[U_{\phi}\right]$ so to prove equality we assume $\phi \in \theta_{0}(\Gamma(X(\mathfrak{V}), S(\mathfrak{U}))), a, b \in A$ such that $\left|\left(r_{a}, r_{b}\right)\right| \subseteq U_{\phi}$ and prove $\left(r_{a}, r_{b}\right) \in \dot{\phi} . \quad\left|\left(r_{a}, r_{b}\right)\right|=\{M \in X(\mathfrak{X}): \quad(a, b,) \notin \bar{M}\} \cong U_{\phi} \quad$ implies that $(a, b) \in \bar{M}$ for every $M \in X(\mathfrak{U})$ such that $\xi_{\mathfrak{\varkappa}}^{-1}(\phi) \in M$. Taking $I$, in Lemma 3.4, as the principal ideal of $\theta_{0}(\mathfrak{U})$ generated by $\xi_{\mathfrak{r}}^{-1}(\phi)$, it is immediate from the above statement that $(a, b) \in \xi_{\mathfrak{q}}^{-1}(\phi)$. Thus, $\left(r_{a}, r_{b}\right) \in \phi$ completing the proof of (iii) and hence the lemma.

We summarize the results from $3.1-3.6$ in the following.

THEOREM 3.7. If $\mathfrak{X}$ is an algebra satisfying conditions (I), (II), then $(X(\mathfrak{U}), S(\mathfrak{U}))$ is a reduced sheaf of algebras such that $\xi_{\mathfrak{x}}$ is an isomorphism from $\mathfrak{A}$ onto $\Gamma(X(\mathfrak{A}), S(\mathfrak{U}))$.

4. Uniqueness. In this section we show, for an algebra $\mathfrak{A}$, that there is at most one reduced sheaf $(X, S)$ (up to isomorphism) for which $\Gamma(X, S) \cong \mathfrak{A}$. Actually we prove more. In Proposition 2.4 we saw that $\Gamma(X, S)$ satisfies conditions (I), (II) whenever $(X, S)$ is reduced; thus, the construction in $\S 3$ yields a reduced sheaf $(X(\Gamma(X, S)), S(\Gamma(X, S)))$ whenever $(X, S)$ is reduced. The desired uniqueness result follows from the fact these two sheaves are isomorphic.

A pair of functions $(\lambda, f)$ is an isomorphism of $(X, S)$ onto $(Y, R)$ if $\lambda$ is a homeomorphism of $X$ onto $Y$ and $f$ is a homeomorphism of $R$ onto $S$ such that $f$ maps $R_{\lambda(x)}$ isomorphically (as an algebra) onto $S_{x}$ for all $x \in X$.

The trivial sheaf provides a special uninteresting case. If $(X, S)$ is trivial, $\Gamma(X, S)$ is a one element algebra; thus, $(X(\Gamma(X, S))$, $S(\Gamma(X, S)))$ is trivially isomorphic to $(X, S)$ and Theorem 4.2 holds in this case. 
Henceforth, we assume $(X, S)$ is a nontrivial reduced sheaf. For $x \in X$ define $\nu_{x}=\theta[X \sim\{x\}]=\left\{(\sigma, \tau) \in{ }^{2} \Gamma(X, S): \sigma_{x}=\tau_{x}\right\} . \quad \nu_{x} \quad$ is a congruence relation on $\Gamma(X, S)$.

Lemma 4.1. (a). For $x \in X$ the map $\sigma \rightarrow \sigma(x)$ is a homomorphism of $\Gamma(X, S)$ onto $S_{x}$ with kernel $v_{x}$.

(b) The map $\lambda: x \rightarrow \gamma\left(v_{x}\right)=I_{\nu_{x}}$ is a homeomorphism of $X$ onto $X(\Gamma(X, S))$.

Proof. (a). is obvious. (b). The last paragraph of $\S 2$ implies $\gamma\left(\prime_{x}\right)$ is a maximal ideal of $\theta_{0}(\Gamma(X, S))$; thus $\lambda$ is a function from $X \rightarrow X(\Gamma(X, S))$. For $x, y \in X, x \neq y$, we can separate $x$ and $y$ by a clopen set ( $X$ is a Boolean space), say $x \in N, y \notin N$ where $N$ is clopen. Therefore $\theta[N] \in \gamma\left(v_{y}\right)$ but $\theta[N] \notin \gamma\left(v_{x}\right)$ so $\gamma\left(v_{x}\right) \neq \gamma\left(v_{y}\right)$ and $\lambda$ is $1-1$.

Now suppose $M \in X(\Gamma(X, S))$. By the correspondence between ideals of $\theta_{0}(\Gamma(X, S))$ and open sets in $X, U_{\bar{H}}$ is a maximal open subset, say $X \sim\{y\}$ for some $y \in X$; thus, $\bar{M}=\theta\left[U_{\bar{M}}\right]=v_{y}$ and $\gamma\left(v_{y}\right)=M$ since $\bar{M}$ is regular. Hence $\lambda$ is onto.

An element of $\theta_{0}(\Gamma(X, S))$ has the form $\theta[N]$ where $N$ is clopen in $X$; thus the sets of the form $\mathscr{N}(\theta[N))$ give a basis for $X(\Gamma(X, S))$. Claim. $\lambda^{-1}(\mathscr{N}(\theta[N]))=N$.

If $x \in X \sim N, N \subseteq X \sim\{x\}$ so $\theta[N] \leqq \theta[X \sim\{x\}]$; thus, $\theta[N] \in \lambda(x)$. Hence $x \in X \sim N$ implies $\lambda x \notin \mathscr{N}(\theta[N])$. Now, if $x \in N, X \sim N \subseteq$ $X \sim\{x\} \quad$ so $\theta[X \sim N] \cong \theta[X \sim\{x\}] ;$ thus, $-\theta[N]=\theta[X \sim N] \in \lambda x$. Hence, $\theta[N] \notin \lambda x$, i.e., $\lambda x \in \mathscr{N}(\theta[N])$. This proves the claim.

The claim shows that $\lambda$ is continuous and open; hence it is a homeomorphism as asserted in 4.1 (b).

TheOREM 4.2. Suppose $(X, S)$ is a reduced sheaf and $\lambda: X \rightarrow X(\Gamma(X, S))$ is defined by $\lambda(x)=\gamma\left(\nu_{x}\right)$ as above. Then there is an $f: S(\Gamma(X, S)) \rightarrow S$ such that $(\lambda, f)$ is an isomorphism of $(X, S)$ onto $(X(\Gamma(X, S)), S(\Gamma(X, S)))$.

Proof. Assume $(X, S)$ is nontrivial. By 4.1 (a) there is an isomorphism $f_{x}$ from $\Gamma(X, S) / \nu_{x}$ onto $S_{x}$ defined by $f_{x}\left(\sigma / v_{x}\right)=\sigma(x)$. Let $f$ be the unique function defined on $S(\Gamma(X, S)$ ) extending all $f_{x}^{\prime} s, x \in X$. It is clear that $f$ is a $1-1$ map of $S(\Gamma(X, S))$ onto $S$ which maps $S(\Gamma(X, S))_{\lambda x}$ isomorphically onto $S_{x}$.

For $\sigma \in \Gamma(X, S)$ and a clopen subset $N$ of $X$ we claim: $\left.f\left(r_{\sigma}(\theta[N])\right)\right)=\sigma(N)$.

From the proof of $4.1(\mathrm{~b}), \quad \mathscr{N}(\theta[N])=\lambda(N)$. Now, for $y \in \mathscr{N}(\theta[N])$, say $y=\lambda x$ for some $x \in N, f\left(r_{\sigma}(y)\right)=f_{x}\left(\sigma / \nu_{x}\right)=\sigma(x) \in \sigma(N)$. Hence $f\left(r_{\sigma}(\mathscr{N}(\theta[N]))\right) \subseteq \sigma(N)$. Now suppose $\sigma(x) \in \sigma(N)$ where $x \in N$. Then $\lambda(x) \in \mathscr{N}(\theta[N])$; so $r_{\sigma}(\lambda x) \in r_{\sigma}(\mathscr{N}(\theta[N]))$ and the above computa- 
tion now shows that $\sigma(x)=f\left(r_{\sigma}(\lambda x)\right) \in f\left(r_{\sigma}(\mathscr{N}(\theta[N]))\right)$. Hence the claim holds.

Since $\{\sigma(N): \sigma \in \Gamma(X, S), N$ clopen subset of $X\}$ forms a basis for $S$ and $\left\{r_{\sigma}\left(\mathscr{N}((\phi)): \sigma \in \Gamma(X, S), \phi \in \theta_{0}(\Gamma(X, S))\right\}\right.$ forms a basis for $S(\Gamma(X, S))$, the claim implies that $f$ gives a $1-1$ correspondence between these two bases. Thus, $f$ is a homeomorphism and it follows that $(\lambda, f)$ is an isomorphism of $(X, S)$ onto $(X(\Gamma(X, S)), S(\Gamma(X, S)))$.

Corollary 4.3. If $(X, S)$ is a reduced sheaf such that $\Gamma(X, S) \cong \mathfrak{A}$, then $(X, S)$ is isomorphic to $(X(\mathfrak{U}), S(\mathfrak{U}))$.

5. Representations with respect to equational classes. Suppose $\mathscr{C}$ is an equational class of algebras. We say that $(X, S)$ is a sheaf of algebras in $\mathscr{K}$ if each stalk is a member of $\mathscr{K}$. Obviously, $\Gamma(X, S)$ belongs to $\mathscr{K}$ whenever $(X, S)$ is a sheaf of algebras in $\mathscr{K}$. By the construction in $\S 3$ each stalk of $(X(\mathfrak{U}), S(\mathfrak{U}))$ is a quotient of $\mathfrak{U}$; thus, $(X(\mathfrak{U}), S(\mathfrak{U}))$ is a sheaf of algebras in $\mathscr{K}$ whenever $\mathfrak{A} \in \mathscr{K}$. As a consequence of 3.7 and 4.3 we have the following result for equational classes.

THEOREM 5.1. Suppose $\mathscr{K}$ is an equational class of algebras such that every member of $\mathscr{K}$ satisfies conditions (I), (II). Then, for every $\mathfrak{X} \in \mathscr{K}$ there is a unique (up to isomorphism) reduced sheaf $(X(\mathfrak{U}), S(\mathfrak{U}))$ of algebras in $\mathscr{K}$ such that $\xi_{\mathfrak{r}}: \mathfrak{U} \cong \Gamma(X(\mathfrak{U}), S(\mathfrak{U}))$.

The above result has appeared in the literature for special choices of $\mathscr{K}$. For $\mathscr{K}$ equal to the class of all rings with unit see R. S. Pierce [3] and for $\mathscr{K}$ equal to the class of all cylindric algebras with a specified dimension see [1]. For special choices of $\mathscr{K}$ the notion of a reduced sheaf takes a simplier form.

\section{REFERENCES}

1. S. D. Comer, A sheaf-theoretic duality theory for cylindric algebras, to appear.

2. K. Keimel, Darstellung von Halbgruppen und universellen Algebren durch Schnitte in Garben; biregulare Halbgruppen, Math. Nachr., to apper.

3. R. S. Pierce, Modules over commutative regular rings., Memoir Amer. Math. Soc., No. $70,1967$.

4. - Introduction to the Theory of Abstract Algebras, Holt. Rinehart and Winston, New York, 1968.

Received July 14, 1970. The results presented in this paper were obtained during investigations supported in part by United States National Science Foundation Grants GP-11804 and GP-21059. The author wishes to thank the referee for his many helpful suggestions. 


\section{PACIFIC JOURNAL OF MATHEMATICS}

\section{EDITORS}

H. SAMELSON

Stanford University

Stanford, California 94305

C. R. Hовву

University of Washington

Seattle, Washington 98105
J. DUGUNDJI

Department of Mathematics

University of Southern California

Los Angeles, California 90007

RICHARD ARENS

University of California

Los Angeles, California 90024

\section{ASSOCIATE EDITORS}
E. F. BECKENBACH
B. H. NeumanN
F. WOLF
K. YoSHIDA

\section{SUPPORTING INSTITUTIONS}

UNIVERSITY OF BRITISH COLUMBIA

CALIFORNIA INSTITUTE OF TECHNOLOGY

UNIVERSITY OF CALIFORNIA

MONTANA STATE UNIVERSITY

UNIVERSITY OF NEVADA

NEW MEXICO STATE UNIVERSITY

OREGON STATE UNIVERSITY

UNIVERSITY OF OREGON

OSAKA UNIVERSITY

UNIVERSITY OF SOUTHERN CALIFORNIA
STANFORD UNIVERSITY

UNIVERSITY OF TOKYO

UNIVERSITY OF UTAH

WASHINGTON STATE UNIVERSITY

UNIVERSITY OF WASHINGTON

AMERICAN MATHEMATICAL SOCIETY CHEVRON RESEARCH CORPORATION NAVAL WEAPONS CENTER 


\section{Pacific Journal of Mathematics}

\section{Vol. 38, No. $1 \quad$ March, 1971}

Bruce Alan Barnes, Banach algebras which are ideals in a Banach algebra ..... 1

David W. Boyd, Inequalities for positive integral operators............... 9

Lawrence Gerald Brown, Note on the open mapping theorem .............. 25

Stephen Daniel Comer, Representations by algebras of sections over Boolean

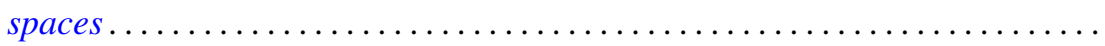

John R. Edwards and Stanley G. Wayment, On the nonequivalence of

conservative Hausdorff methods and Hausdorff moment sequences ........

P. D. T. A. Elliott, On the limiting distribution of additive functions $(\bmod 1) \ldots \ldots$

Mary Rodriguez Embry, Classifying special operators by means of subsets

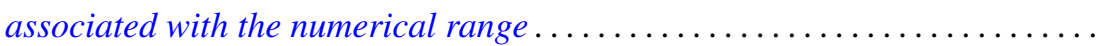

Darald Joe Hartfiel, Counterexamples to a conjecture of G. N. de Oliveira ......

C. Ward Henson, A family of countable homogeneous graphs...............

Satoru Igari and Shigehiko Kuratsubo, A sufficient condition for

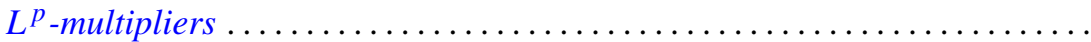

William A. Kirk, Fixed point theorems for nonlinear nonexpansive and

generalized contraction mappings............................

Erwin Kleinfeld, A generalization of commutative and associative rings ...... 95

D. B. Lahiri, Some restricted partition functions. Congruences modulo $11 \ldots \ldots 103$

T. Y. Lin, Homological algebra of stable homotopy ring $\pi *$ of spheres ....... 117

Morris Marden, A representation for the logarithmic derivative of a meromorphic function...........................

John Charles Nichols and James C. Smith, Examples concerning sum properties for metric-dependent dimension functions . .

Asit Baran Raha, On completely Hausdorff-completion of a completely

Hausdorff space.

M. Rajagopalan and Bertram Manuel Schreiber, Ergodic automorphisms and affine transformations of locally compact groups..........

N. V. Rao and Ashoke Kumar Roy, Linear isometries of some function

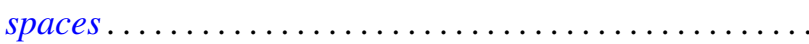

William Francis Reynolds, Blocks and F-class algebras of finite groups

Richard Rochberg, Which linear maps of the disk algebra are multiplicative ...

Gary Sampson, Sharp estimates of convolution transforms in terms of decreasing

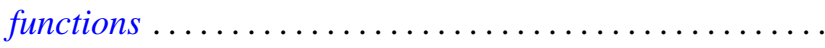

Stephen Scheinberg, Fatou's lemma in normed linear spaces

Ken Shaw, Whittaker constants for entire functions of several complex

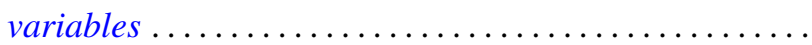

James DeWitt Stein, Two uniform boundedness theorems................ 251

$\mathrm{Li} \mathrm{Pi} \mathrm{Su,} \mathrm{Homomorphisms} \mathrm{of} \mathrm{near-rings} \mathrm{of} \mathrm{continuous} \mathrm{functions} \mathrm{.} \mathrm{.............} 261$

Stephen Willard, Functionally compact spaces, $C$-compact spaces and mappings of minimal Hausdorff spaces....................... 